\title{
Evolution of soap froth under temperature effects
}

\author{
W.Y. Tam and K.Y. Szeto \\ Department of Physics, The Hong Kong University of Science and Technology, Clear Water Bay, Kowloon, Hong Kong
}

(Received 26 July 1995)

\begin{abstract}
Experiments on two-dimensional soap froth under temperature ramping have been performed. At constant temperatures, the classical linear dependence of the averaged cell area with time is observed at different temperatures. The rate of change of area depends sensitively on temperature. For froths under constant temperature ramping, in contrast to the classical results, a quadratic evolution of the average cell area is observed. This can be explained by a linear dependence of the diffusion rate on temperature.
\end{abstract}

PACS number(s): 82.70.Rr, 68.90.+g

\section{INTRODUCTION}

During the past ten years the dynamics of evolving soap foams has become a topic of increasing interest [1]. Although the structure of such foams has been recognized for some time to provide valuable models for structures ranging from biological tissue to metallic grain boundaries [2], it is only recently that detailed experimental observations [3-7] and numerical simulations [8-11] have become available. The present understanding is that diffusion and topological processes are the main driving forces for the coarsening of soap froths. The famous von Neumann law [12], which predicts that an $n$-sided bubble will grow for $n>6$ or shrink for $n<6$ with a linear time dependence, has been verified both in experiments and in numerical simulations. However, these experiments were conducted mostly at room temperature. The temperature dependence of the diffusion rate was also neglected in theoretical and numerical studies because much of these efforts have been devoted to the verification of certain dynamical scaling laws $[5,13,14]$. A recent study of breaking soap foams due to thermal stress has raised interest in temperature-dependent diffusion and its effects on the dynamics of soap foams [15]. We report experimental results of two-dimensional soap froth in which the temperature of the froth is fixed at constant values or varied with a constant ramping rate. We find a linear time dependence for constant temperature froth but a nonlinear time dependence for the temperature ramped froth.

\section{EXPERIMENT}

The bubble chamber consists of two 24-cm-diam circular glass plates separated by a ring spacer of $0.16 \mathrm{~cm}$ thickness. Soap solution and air are introduced into the gap between the plates from inlets through the spacer. The whole setup is enclosed in another chamber containing circulating water from a temperature bath, which is controlled by a personal computer. A dark field imaging method is employed using a circular fluorescence lamp mounted above the setup. Images of the two-dimensional froth at the central region of the chamber are captured by a charge coupled device camera with $768 \times 575$ pixel resolution.

The soap solution used in the experiment is $0.1 \mathrm{M}$ of NaDS in distilled water. To prepare the soap froth a fixed amount of the soap solution was first injected into the bubble chamber. Then bubbles generated from an external reservoir containing the same solution were pumped into the chamber. This method produces bubbles with random sizes and shortens the waiting time to the scaling state. Appropriate release of bubbles through an outlet in the spacer is performed to obtain a uniform quasi-twodimensional froth. Then the outlet was closed while bubbles were continually pumped into the chamber from the inlet for a short time to create a slightly pressurized froth. To remove excess soap fluid, the froth was first drained vertically with the inlet at the lowest position for a fixed period and then the chamber was released to atmospheric pressure by opening the inlet. After removing the excess fluid, the chamber was sealed by closing the inlet and outlet. The resulted froth was dry with a Plateau border less than $0.3 \mathrm{~mm}$ and no changes were observed for the duration of experiment. The above procedures were crucial for consistency in all the runs as gas diffusion between bubbles depends very much on the thickness of the boundaries and the Plateau borders [16]. The temperature of the froth was held at $20^{\circ} \mathrm{C}$ during the preparation. For constant temperature froth, the temperature was ramped for $3 \mathrm{~h}$ to the desired values and was held fixed for the duration of the experiment. The froth equilibrated into the scaling state after the ramping period. The experiment for constant temperature was repeated several times at each temperature from $20^{\circ} \mathrm{C}$ to $32^{\circ} \mathrm{C}$ for better statistics. It was difficult to obtain satisfactory data at higher temperatures due to breakage of wall boundaries for the soap used. For temperature ramped froth, the temperature was held constant at $20^{\circ} \mathrm{C}$ for $3 \mathrm{~h}$ before the ramping procedure. All samples were prepared with the same waiting period to ensure that all ramping experiments started with a scaling state as initial conditions. We chose the start of the ramping as the time 


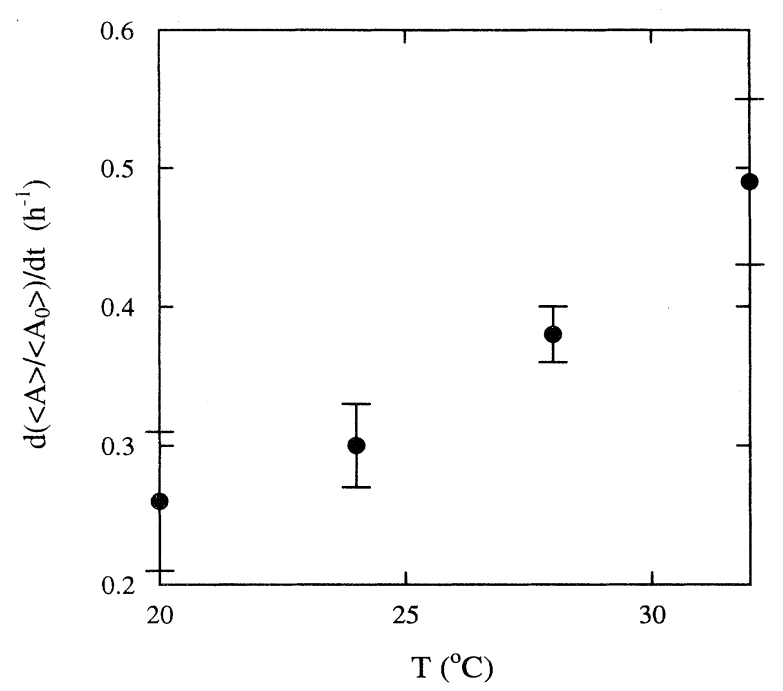

FIG. 1. Rate of growth of the soap froth normalized area $\langle A\rangle /\left\langle A_{0}\right\rangle$ versus temperature $T$.

origin and the temperature was increased with a constant ramping rate $\alpha$. Each run lasted for a few hours to a few days, depending on the ramping rate, and was stopped when wall rupture started. It was observed that wall rupture occurred at about $35^{\circ} \mathrm{C}-45^{\circ} \mathrm{C}$, depending on the ramping rate. Again, several runs for each ramping rate were carried out and consistency between runs was obtained. In this paper, we present only the data taken without wall rupture.

At constant temperature, the averaged cell area is found to increase linearly with time in agreement with the well established results $[1,6]$ for all temperatures used. Since at constant temperature, we have data in agreement with von Neumann's law, we show in Fig. 1 the rate of change of the average cell area $\langle A\rangle$ normalized by

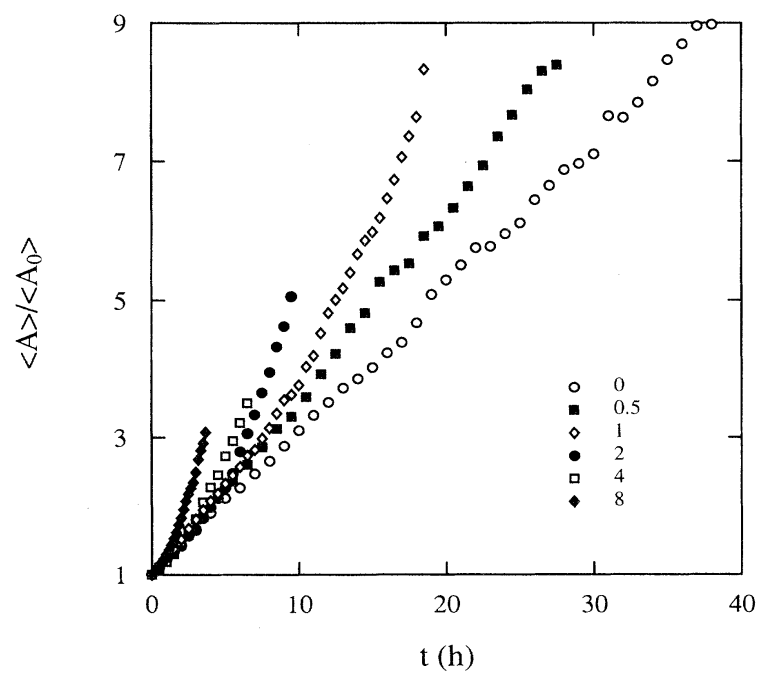

FIG. 2. Growth of the soap froth normalized area $\langle A\rangle /\left\langle A_{0}\right\rangle$ versus time $t$ for six different ramping rate $\alpha=0,0.5,1,2,4,8$ $\mathrm{K} / \mathrm{h}$. the initial average cell area $\left\langle A_{0}\right\rangle$ as a function of temperature. The error bars are standard derivations obtained from three to four runs taken at the same temperature. The data show a strong dependence upon temperature, as expected.

Figure 2 shows the averaged area $\langle A\rangle$ at $t=0$ for typical runs using ramping rates $\alpha=0,0.5,1.0,2.0,4.0$, and $8.0 \mathrm{~K} / \mathrm{h}$, as indicated by the different symbols. In the case of high ramping rates, wall rupture occurs sooner, thus limiting the number of data points in the figure. We see from Fig. 2 that the normalized area increased faster than linearly for all ramping rates, except for $\alpha=0$, which is linear in time. Our results indicate a direct correspondence between the coarsening of the froth and the ramping rate. Despite the nonlinear dependence of time, we find that there are scalings in the side and the area distributions even for runs with $\alpha>0 \mathrm{~K} / \mathrm{h}$. This is seen from the distribution functions $f(n)$ and $f(A)$ shown in Fig. 3. However, when there is wall rupture,
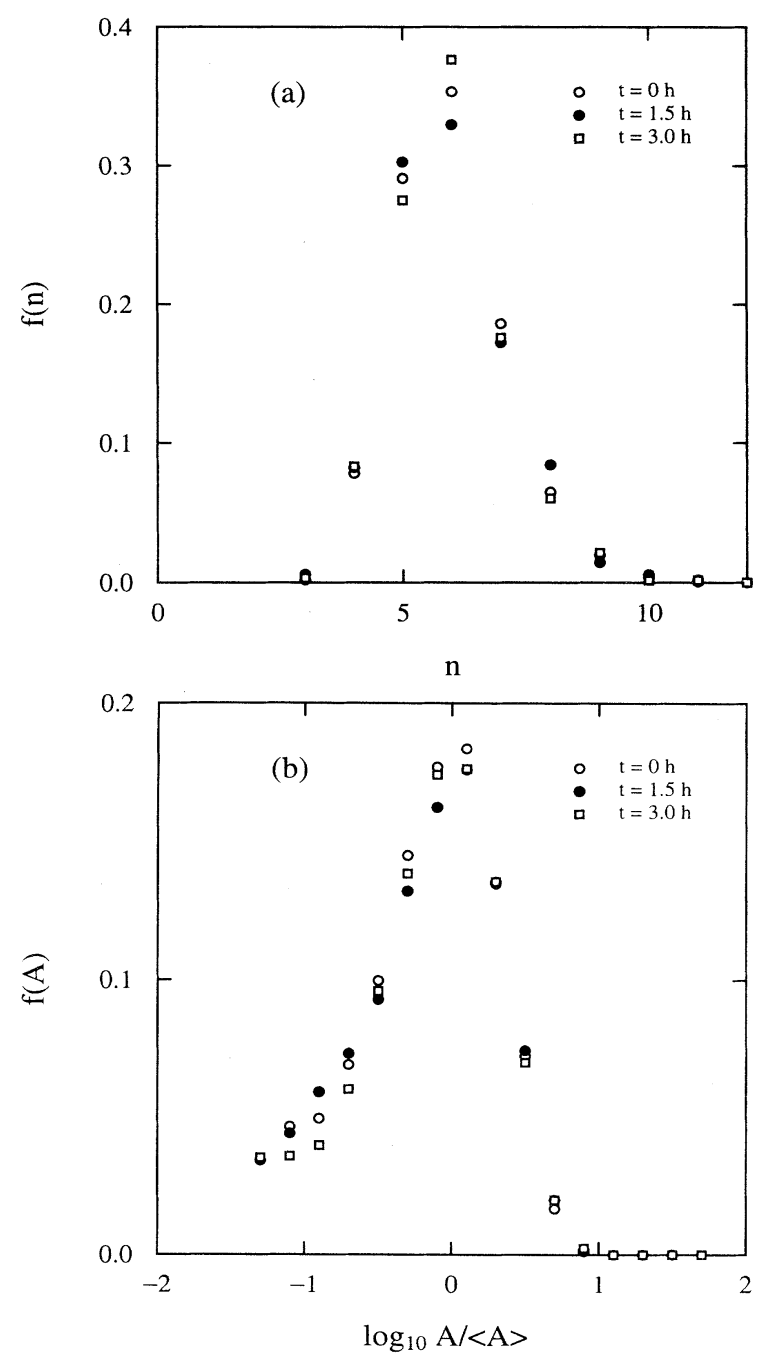

FIG. 3. (a) Distribution function vs the number of sides of the cell for $\alpha=8 \mathrm{~K} / \mathrm{h}$. (b) Distribution function vs logarithm of the normalized area for $\alpha=8 \mathrm{~K} / \mathrm{h}$. 
scalings no longer hold, in agreement with a recent study $[15]$.

\section{NONLINEAR EVOLUTION}

The original derivation of von Neumann's law for twodimensional froth assumes that the gas diffusion across the bubble wall occurs at contant temperature. The pressure difference between adjacent bubbles drives this diffusion flow and the area change of a particular bubble will be linearly proportional to the amount of gas flow to and from its neighbors. When the temperature of the system changes, the diffusion constant that characterizes the flow will also change. This will introduce nonlinear correction to the evolution equation of the area. Since the scaling behavior is unaffected by temperature ramping, we assume that the fraction of $n$-sided cells $f(n)$ in the soap froth is independent of time. The mean area of the bubble grows linearly with time because of the disappearance of three-, four-, and five-sided bubbles. Thus, if $N$ is the total number of bubbles in a sample area $A_{\text {tot }}$, we have

$$
\begin{aligned}
\frac{d N}{d t} & =\sum_{n=3,4,5} N_{n} \frac{1}{A_{n}} \frac{d A_{n}}{d t} \\
& =\sum_{n=3,4,5} f(n) N \frac{\kappa(n-6)}{A_{n}} \\
& =-N^{2} \sum_{n=3,4,5} f(n) \frac{\kappa(6-n)}{x_{n} A_{\text {tot }}} .
\end{aligned}
$$

Here $\kappa$ is the diffusion constant and $x_{n}=A_{n} /\langle A\rangle$. In the second equality, we make use of $N_{n}=f(n) N$ for the number of $n$-sided bubbles and also von Neumann's law,

$$
\frac{d A_{n}}{d t}=\kappa(n-6) \text {. }
$$

In the last equality of Eq. (1), we see that $x_{n}$ is time independent in the scaling state so that the rate of change of $N$ is proportional to $N^{2}$. Since $\langle A\rangle=A_{\text {tot }} / N$, we have $d\langle A\rangle / d t=d N^{-1} / d t=-\frac{1}{N^{2}} d N / d t=\kappa C$, where $C$ is a positive constant. Thus $N \approx t^{-1}$ and $\langle A\rangle=A_{\text {tot }} / N \approx t$.

If we assume that the temperature ramping only affects the diffusion constant $\kappa$, not the validity of von Neumann's law, then a substitution of the appropriate $\kappa$ at different temperatures will yield the correct evolution of $\langle A\rangle$. Based on this assumption, the nonlinear evolution of the soap froth when the ramping rate is nonzero can be explained simply by combining the information in Fig. 1 about the rate of area growth vs temperature with data on experiment with constant ramping rate.

Let us assume a linear fit to Fig. 1,

$$
\frac{1}{\left\langle A_{0}\right\rangle} \frac{d\langle A\rangle}{d t}=a T+b \propto \kappa .
$$

Then, for constant ramping rate, we have $T(t)=T_{0}+\alpha t$, where $T_{0}=293 \mathrm{~K}$ is the initial temperature and $\alpha$ is the ramping rate. By integrating Eq. (3) with time using $T=T(t)$, we get

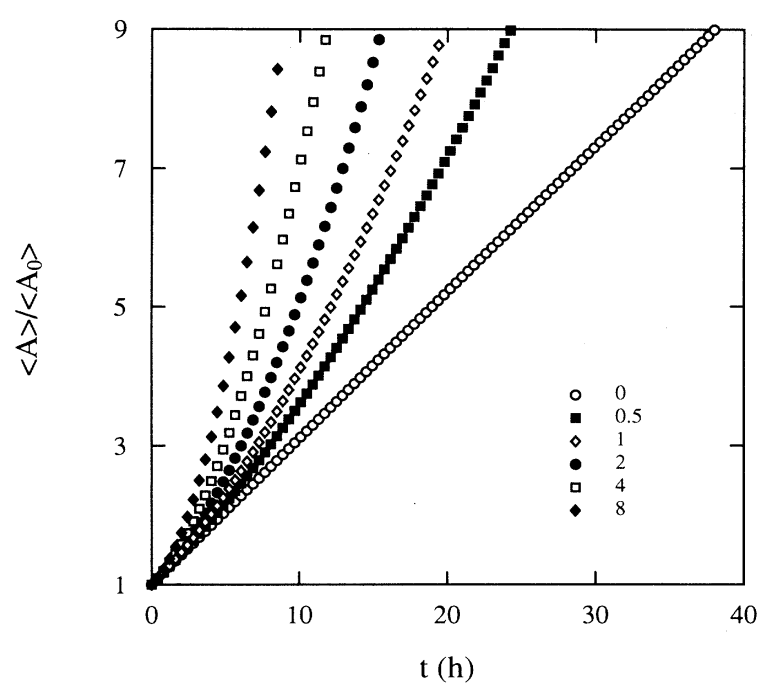

FIG. 4. Theoretical calculation of the soap froth normalized area $\langle A\rangle /\left\langle A_{0}\right\rangle$ versus time $t$ for six different ramping rates $\alpha=0,0.5,1,2,4,8 \mathrm{~K} / \mathrm{h}$.

$$
\frac{\langle A\rangle}{\left\langle A_{0}\right\rangle}=1+\left(a T_{0}+b\right) t+\frac{1}{2} a \alpha t^{2},
$$

with $\alpha$ in $\mathrm{K} / \mathrm{h}$ and $t$ in h. For $\alpha=0$, we have a linear fit to the experimental data in Fig. 2 to get $a T_{0}+b=$ $0.2105 \mathrm{~h}^{-1}$. With this constraint, we return to Fig. 1 to fit a linear relation for Eq. (3) and obtain $a \approx 0.0196$ $(\mathrm{Kh})^{-1}$ and $b \approx-5.53 \mathrm{~h}^{-1}$. This is better than a direct fit of Fig. 1 by a straight line as the error bars are large. Equation (4) is plotted in Fig. 4 for the experimental values of $\alpha$ and a comparison with Fig. 2 shows excellent agreement.

\section{DISCUSSION}

Our experimental results on froth with temperature ramping show a departure from the linear evolution of the soap froth area. This nonlinear evolution can be easily obtained by assuming the validity of von Neumann's law at each temperature, with the appropriate diffusion constant $\kappa(T)$ obtained from measurements at several constant temperatures. There are two suggested ciirections for future studies. The first involves an application of data analysis in the examination of scaling relation between the number of neighbors and average size. If $n_{i}$ is the number of neighbors of the $i$ th cell, the scaling relation of the area and perimeter of the cell with $n_{i}$ can be tested for experiments with nonzero ramping rate, thereby testing the validity of the theoretical predictions of Lewis [17] and Feltham [18], which address froth at constant temperature. A second direction involves the scaling relation between the critical temperature $T_{c}$, at which bursting occurs, and the ramping rate. An operational definition of the critical temperature can be given as follow. From the temporal evolution of $\langle A\rangle$, two time regimes can be noted: (i) the 
initial time regime where $\langle A\rangle$ follows the quadratic time law [Eq. (4)] and (ii) the bursting time regime where $\langle A\rangle$ increases rapidly. The crossover of these two regimes defines a crossover time $t^{\star}$, which corresponds to a critical temperature $T_{c}=T_{0}+\alpha t^{\star}$. Since the ramping rate not only determines the temperature at which bursting takes place but also modifies the parameters determining the microscopic process of bursting, we expect that there is some dependence of $t^{\star}$ on $\alpha$. Hence the dependence of the critical temperature on $\alpha$ is expected to be nonlinear and is currently under investigation.

\section{ACKNOWLEDGMENTS}

W. Y. Tam acknowledges support from the Alfred P. Sloan Foundation and the Direct Allocation Grant of HKUST. The authors have enjoyed discussions with $M$. Tabor, Rita M. C. de Almedia, J. J. Chae, and D. Burnett.
[1] A. G. Glazier and D. Weaire, J. Phys. Condens. Matter 4, 1867 (1992).

[2] D. Weaire and N. River, Contemp. Phys. 25, 59 (1984).

[3] J. A. Glazier and J. Stavans, Phys. Rev. A 40, 7398 (1989).

[4] J. A. Glazier, S. P. Gross, and J. Stavans, Phys. Rev. A 36, 306 (1987).

[5] J. Stavans and J. A. Glazier, Phys. Rev. Lett. 62, 1318 (1989).

[6] J. Stavans, Phys. Rev. A 42, 5049 (1990).

[7] J. Stavans, Physica A 194, 307 (1993).

[8] J. A. Glazier, M. P. Anderson, and G. S. Grest, Philos. Mag. B 62, 615 (1990).

[9] E. A. Holm, J. A. Glazier, D. J. Srolovitz, and G. S. Grest, Phys. Rev. A 43, 2662 (1991).
[10] T. Herdtle and H. Aref, J. Fluid Mech. 241, 233 (1992).

[11] J. Wejchert, D. Weaire, and J. P. Kermode, Philos. Mag. B 53, 15 (1986).

[12] J. von Neumann, Metal Interfaces (American Society of Metals, Cleveland, 1952), p. 108.

[13] J. Stavens, E. Domany, and D. Mukamel, Europhys. Lett. 15, 479 (1991).

[14] H. Flyvberg, Phys. Rev. E 47, 4037 (1993); Physica A 194, 298 (1993).

[15] D. Burnett, J. J. Chae, W. Y. Tam, R. M. C. de Almeida, and M. Tabor, Phys. Rev. E 51, 5788 (1995).

[16] F. Bolton and D. Weaire, Phys. Rev. Lett. 65, 3449 (1990); Philos. Mag. B 63, 795 (1991).

[17] D. Lewis, Anat. Rec. 38, 351 (1928).

[18] P. Feltham, Acta Metall. 5, 97 (1957). 